www.jmscr.igmpublication.org

Impact Factor 5.84

Index Copernicus Value: 71.58

ISSN (e)-2347-176x ISSN (p) 2455-0450

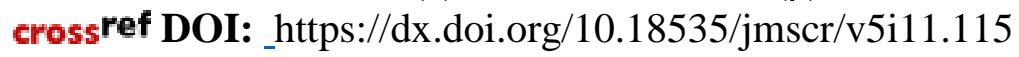

Journal Of Medical Science And Clinical Research

\title{
Coronary Artery Disease Severity Assessment Using Urinary Dipstick
}

\section{Albumin Test}

Authors

\section{Venkatram Murugesan, Nagarajan Kinathankaraiyan, Ashida S Thulaseedharan, Badrinath A Kuppuswamy}

Sri Manakula Vinayagar Medical College and Hospital, Kalitheerthalkuppam, Puducherry 605107 India

Corresponding Author

Venkatram Murugesan

Department of General Medicine, Sri Manakula Vinayagar Medical College and Hospital,

Kalitheerthalkuppam, Puducherry 605107

9042846842, Email: drvenkatram@gmail.com

\begin{abstract}
Coronary artery disease refers to the gradual or sudden, reversible or irreversible obstruction to the heart's blood supply due to the plaque build-up over several years or due to vasospasm. Albuminuria is one of the recently added risk factors for coronary artery disease. Consensus on albuminuria as an individual risk factor and whether it correlates with the severity of the disease is lacking. This study aims to correlate the severity of obstruction of coronary arteries with albuminuria in acute coronary syndrome patients. This study was done to correlate the severity of angiographically proven coronary artery disease with urinary dipstick albumin excretion in acute coronary syndrome patients. Acute coronary syndrome patients with more than 50\% obstruction of one or more coronary arteries demonstrated by conventional coronary angiogram were included. Albuminuria by urinary dipstick method was analyzed. The data obtained was entered in the MS excel sheet and data analysis done using SPSS v24.0. In the study none of the subjects had history of Diabetes, Hypertension, Urinary tract infection and drug history. $65.5 \%$ of the patients had single vessel disease, $14.5 \%$ had double vessel disease and $20 \%$ had triple vessel disease. There was a statistically significant correlation between severity of coronary artery disease and albuminuria.
\end{abstract}

Key Words: Albuminuria, dipstick, coronary artery disease severity, acute coronary syndrome.

\section{Introduction}

Coronary artery disease refers to the gradual or sudden, reversible or irreversible obstruction to the heart's blood supply due to the plaque build-up over several years or due to vasospasm. Presently, though several bio markers for coronary artery disease exist, none can quantify the severity of obstruction of coronary arteries in the form of number of coronary arteries involved. The only detail revealed by them is about the presence or absence of coronary artery disease. Albuminuria is one of the recently added risk factors for coronary artery disease. Consensus on albuminuria as an individual risk factor and whether it correlates with the severity of the disease is lacking.

\section{Aims and Objectives}

This study aims to correlate the severity of obstruction of coronary arteries with albuminuria in acute coronary syndrome patients. 


\section{Materials and Methods}

It is a hospital based, retrospective, cross section study done at a tertiary referral centre in Pondicherry. Patients diagnosed with acute coronary syndrome as defined by the ACC/ESC criteria, who underwent conventional coronary angiogram that demonstrated more than $50 \%$ obstruction of one or more coronary arteries and who had urinary dipstick albumin assay done were included. Patients with diabetes, hypertension, renal disease, urinary tract infection and drug history of angiotensin converting enzyme inhibitors or angiotensin receptor blockers were excluded. The sample size was calculated from the reference study Albuminuria during acute coronary syndrome: A predictor of short term mortality and prognosis by Prahalad Karki et al as 55. This study was done after getting the institute research committee approval. Permission to access the case sheets from the medical records department was obtained from the institute's Medical Superintendent.

\section{Results}

After applying the inclusion and exclusion criteria, the case sheets of patients were retrospectively analyzed from the most recent admission, backwards, till the desired sample size was obtained. A total of 55 subjects fulfilling the criteria were randomly selected.

Table 1: Profile of subjects in the study

\begin{tabular}{|c|c|c|c|}
\hline \multirow{4}{*}{} & <40 years & Count & Percentage \\
\cline { 2 - 4 } & 41 to 50 years & 14 & $10.9 \%$ \\
\cline { 2 - 4 } Age & 51 to 60 years & 19 & $34.5 \%$ \\
\cline { 2 - 4 } & 61 to 70 years & 13 & $23.6 \%$ \\
\cline { 2 - 4 } & >70 years & 3 & $5.5 \%$ \\
\cline { 2 - 4 } & $\begin{array}{c}\text { Mean } \pm \text { Standard } \\
\text { Deviation (years) }\end{array}$ & \multicolumn{2}{|c|}{$54.18 \pm 11$} \\
\hline \multirow{3}{*}{ Gender } & Female & 12 & $21.8 \%$ \\
\cline { 2 - 4 } & Male & 43 & $78.2 \%$ \\
\hline
\end{tabular}

Figure 1: Bar diagram showing Angiographic findings among subjects.

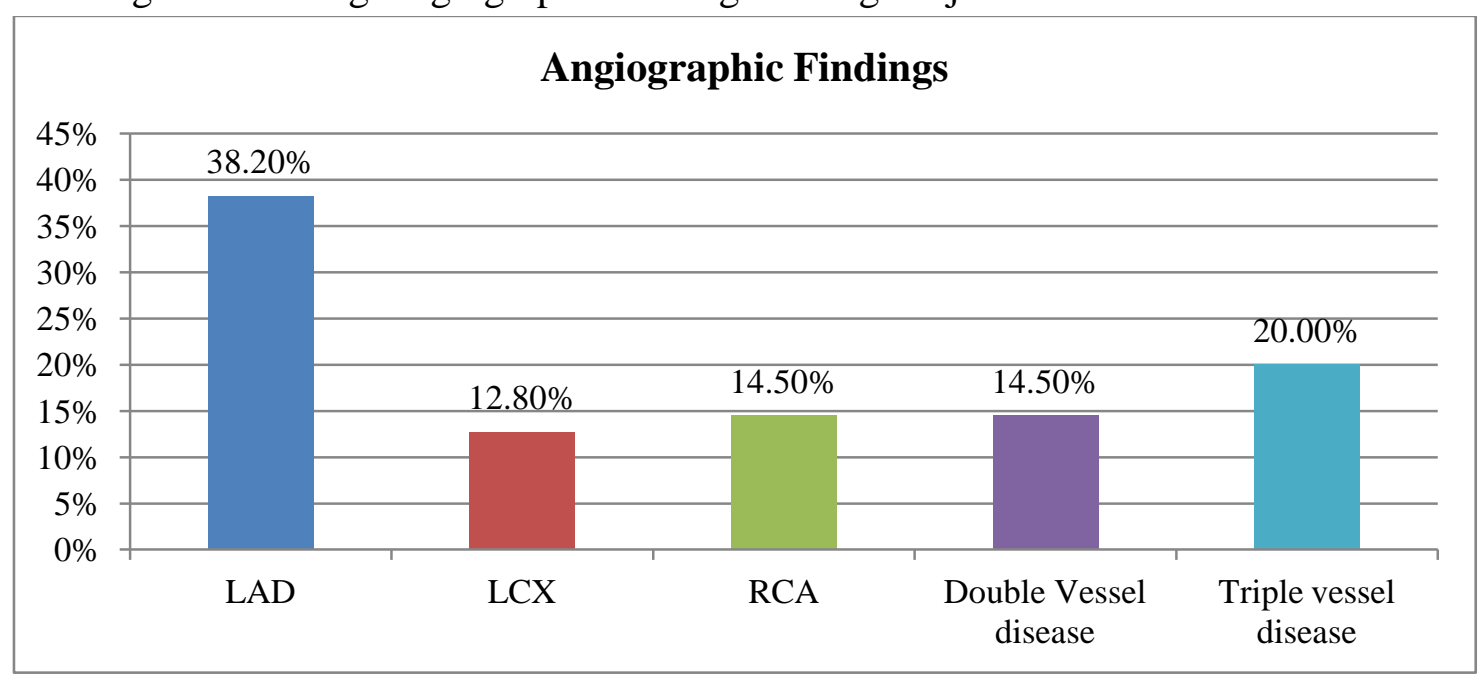

Table 2: Association between Albumin and Angiographic findings

\begin{tabular}{|c|l|c|c|c|c|c|c|}
\hline \multicolumn{2}{|c|}{} & \multicolumn{7}{c|}{ Angiographic Findings } \\
\cline { 3 - 8 } \multicolumn{2}{c|}{} & \multicolumn{2}{|c|}{ Single Vessel } & \multicolumn{2}{c|}{ Double Vessel disease } & \multicolumn{2}{c|}{ Triple vessel disease } \\
\cline { 2 - 8 } & Count & $\%$ & Count & $\%$ & Count & $\%$ \\
\hline \multirow{3}{*}{ Albumin } & Nil & 1 & $2.8 \%$ & 0 & $0.0 \%$ & 0 & $0.0 \%$ \\
\cline { 2 - 8 } & Trace & 1 & $2.8 \%$ & 1 & $12.5 \%$ & 1 & $9.1 \%$ \\
\cline { 2 - 8 } & + & 1 & $2.8 \%$ & 2 & $25.0 \%$ & 0 & $0.0 \%$ \\
\cline { 2 - 8 } & ++ & 25 & $69.4 \%$ & 1 & $12.5 \%$ & 1 & $9.1 \%$ \\
\cline { 2 - 8 } & +++ & 8 & $22.2 \%$ & 4 & $50.0 \%$ & 8 & $72.7 \%$ \\
\cline { 2 - 8 } & ++++ & 0 & $0.0 \%$ & 0 & $0.0 \%$ & 1 & $9.1 \%$ \\
\hline
\end{tabular}

$\chi 2=27.86, \mathrm{df}=10, \mathrm{p}=0.002^{*}$ 
In the study $65.5 \%$ had Single vessel disease, $14.5 \%$ had Double vessel disease and 20\% had Triple vessel disease. In the study there was no significant association between Angiographic findings and age, gender. None of the subjects had history of Diabetes, Hypertension, Urinary tract infection and drug history.

In Single vessel disease, $2.8 \%$ had traces, $2.8 \%$ had $1+, 69.4 \%$ had $2+, 22.2 \%$ had $3+$ albumin. In double vessel disease $12.5 \%$ had traces, $25 \%$ had $1+, 12.5 \%$ had $2+, 50 \%$ had $3+$ and in triple vessel disease $9.1 \%$ had traces, $9.1 \%$ had $2+, 72.7 \%$ had $3+$ and $9.1 \%$ had $4+$. This observation between angiographic findings and albumin was statistically significant. I.e. with increase in no of vessels involved in CAD subjects there was increase in albumin levels.

\section{Discussion}

The prevalence of coronary artery disease is increasing with projections estimating it to be the leading cause of death worldwide by $2020^{1}$. $80 \%$ of the world's death from cardiovascular disease worldwide and $87 \%$ of related disability currently occur in low and middle income countries. In India, the prevalence is more in the low income groups and in rural population ${ }^{2}$. With the increasing incidence of coronary artery disease and its associated morbidity and mortality, it is mandatory to properly stratify the disease based on its severity so that appropriate and timely management strategies can be adopted.

Several studies have demostrated albuminuria as a risk factor for acute coronary syndrome, the likely link postulated to be due to endothelial dysfunction, chronic low-grade inflammation, or increased transvascular leakage of macromolecules ${ }^{3,4}$. Several studies have also demonstrated worse outcomes in patients with acute coronary syndrome who also had albuminuria to their non-albuminuric counterparts. However, studies on whether it can predict the severity of disease have not yet been done.

This study aims at correlating the severity of obstruction of angiographically proven coronary artery disease with albuminuria demonstrated by urinary dipstick method in acute coronary syndrome patients. Urinary dipstick method is fast, economical and can be performed easily. Urinary albumin creatinine ratio was not assessed because of the above mentioned reasons. Also, because this study aims at helping the health care facilities with limited resources to better explain the prognosis of the patients and in prompt referral of acute coronary syndrome patients to appropriate centres for carrying out coronary intervention procedures without wasting much time and money.

In the study, the prevalence of albuminuria was 98.2\% which was much higher compared to other studies where it is around $30-40 \%$ despite the fact that other common causes for albuminuria were excluded $^{3,4,5,6}$. This may be due to the fact that all the samples were taken on the day of admission while it was taken three days or later in other studies, and also the sample size was only 55 which is much lesser and thus may not be predictive of a community level prevalence. Karki et al in their study had a prevalence of $87 \%$ which, they attributed to the same reason. This study focused on correlating the severity of obstruction of coronary arteries, and thus the type of presentation of acute coronary syndrome was not assessed.

The average age of presentation was 54.18 years and males accounted for $78.2 \%$ of the study group. None of the patients had diabetes or hypertension which makes this study unique. $65.5 \%$ of the patients had single vessel disease and Left anterior descending artery was the most commonly involved, contributing $38.2 \%$.

It was observed that the number of vessels involved was associated with an incremental increase in the amount of albumin excreted and this was statistically significant. Previous studies had concluded that the clinical outcomes were poorer in the albuminuric group as compared to the non albuminuric counterparts ${ }^{7,8,9}$, however, none of the studies demonstrated the relationship between the amount of albuminuria and the severity of obstruction of coronary arteries despite the fact 
that they used albumin creatinine ratio in the place urine spot albumin assay ${ }^{10}$. In acute coronary syndrome patients, if the other causes for albuminuria can be excluded, urinary dipstick albumin may be a useful tool to assess the likely severity of obstruction of coronary artery occlusion.

\section{Conclusion}

Spot urinary dipstick albumin can be used to assess the likely severity of obstruction of coronary arteries provided other causes for albuminuria are excluded.

\section{Reference}

1. Reddy KS. Cardiovascular disease in nonWestern countries. New England Journal of Medicine. 2004 Jun 10;350(24):2438-40.

2. Reddy KS, Yusuf S. Emerging epidemic of cardiovascular disease in developing countries. Circulation. 1998 Feb 17;97(6):596-601.

3. Karki P, Shrestha H, Shrestha NR. Albuminuria during acute coronary syndrome: A predictor for short term mortality and prognosis. Journal of Indian College of Cardiology. 2014 Sep 30;4(3):139-43.

4. Weir MR. Microalbuminuria and cardiovascular disease. Clinical Journal of the American Society of Nephrology. 2007 May 1;2(3):581-90.

5. Hoseini VN, Rasouli M. Microalbuminuria correlates with the prevalence and severity of coronary artery disease in non-diabetic patients. Cardiology journal. 2009;16(2): 142-5.

6. Pradhep RS, Janakiram N, Benjamin VP, Natarajan S. A Study of Microalbuminuria in Coronary Artery Disease among NonDiabetic Individuals. Int. J. Curr. Microbiol. App. Sci. 2017;6(1):344-61.

7. Rein P, Saely $\mathrm{CH}$, Vonbank A, Fraunberger $\mathrm{P}$, Drexel H. Is albuminuria a myocardial infarction risk equivalent for atherothrom- botic events?. Atherosclerosis. 2015 May 31;240(1):21-5.

8. Bouchi R, Babazono $\mathrm{T}$, Yoshida N, Nyumura I, Toya K, Hayashi T, Hanai K, Tanaka N, Ishii A, Iwamoto Y. Association of albuminuria and reduced estimated glomerular filtration rate with incident stroke and coronary artery disease in patients with type 2 diabetes. Hypertension Research. 2010 Dec 1;33(12):1298-304.

9. Escobedo J, Rana JS, Lombardero MS, Albert SG, Davis AM, Kennedy FP, Mooradian AD, Robertson DG, Srinivas VS, Gebhart SS, BARI 2D Study Group. Association between albuminuria and duration of diabetes and myocardial dysfunction and peripheral arterial disease among patients with stable coronary artery disease in the BARI 2D study. InMayo Clinic Proceedings 2010 Jan 31 (Vol. 85, No. 1, pp. 41-46). Elsevier.

10. Maahs DM, Snell-Bergeon JK, Kinney GL, Wadwa RP, Garg S, Ogden LG, Rewers M. ACE-I/ARB treatment in type 1 diabetes patients with albuminuria is associated with lower odds of progression of coronary artery calcification. Journal of diabetes and its complications. 2007 Oct 31;21(5):273-9. 